\title{
The Value of Serum Amyloid A in the Diagnosis and Management of Ankylosing Spondylitis
}

\section{Qi-Lei Hu \\ Shui Fu \\ Rong Huang \\ Liang Zhang \\ Li-Feng Wu \\ Yin-Jiang Lv}

Department of Clinical Laboratory, The First People's Hospital of Yuhang District, Hangzhou, Hangzhou, Zhejiang, 31 I I00,

People's Republic of China
Correspondence: Yin-Jiang Lv Department of Clinical Laboratory, The First People's Hospital of Yuhang District, Hangzhou, No. 369 Yingbin Road of Nanyuan Street, Yuhang District, Hangzhou, 31 I100, People's Republic of China

Tel +8657I 89369404

Email Lvyj6217@I63.com
Objective: The present study aimed to explore the clinical value of serum amyloid A (SAA) in the diagnosis, treatment, and assessment of ankylosing spondylitis (AS).

Methods: Seventy-eight patients with AS were enrolled as the case group, while the control group consisted of 80 healthy individuals enrolled during the same time period. According to the criteria of the Bath Ankylosing Spondylitis Disease Activity Index (BASDAI), patients in the case group were divided into those in the remission phase (36 patients) and those in the active phase (42 patients). Levels of SAA, C-reactive protein (CRP), and erythrocyte sedimentation rate (ESR) were measured in all enrolled subjects and analyzed.

Results: SAA levels were significantly higher in the AS group $(39.65 \pm 12.32 \mathrm{ng} / \mathrm{mL})$ than in the control group $(7.64 \pm 1.32 \mathrm{ng} / \mathrm{mL})(\mathrm{p}=0.011)$ and in patients in the active phase $(56.18$ $\pm 17.25 \mathrm{ng} / \mathrm{mL})$ compared with those in the remission phase $(20.36 \pm 5.36 \mathrm{ng} / \mathrm{mL})(\mathrm{p}$ $=0.015$ ). The sensitivity and specificity of SAA were $79.49 \%$ and $77.50 \%$, respectively. There was a positive correlation between SAA level and the BASDAI grade $(r=0.77$, $\mathrm{p}=0.005)$, CRP level $(\mathrm{r}=0.68, \mathrm{p}=0.011)$, and ESR $(\mathrm{r}=0.62, \mathrm{p}=0.012)$.

Conclusion: Not only is SAA a reliable indicator for the presence of AS, it may also be useful for monitoring the activity of this disease.

Keywords: ankylosing spondylitis, serum amyloid A, C-reactive protein, erythrocyte sedimentation rate, disease activity

\section{Introduction}

Ankylosing spondylitis (AS) is an autoimmune disease of unknown etiology, characterized by insidious onset, a chronic inflammatory response, atypical symptoms, and repeated and continuous progression of the disease. It can seriously impact the physical and mental health, and quality of life of patients. ${ }^{1-3}$ Due to the rapid progression of the disease, early diagnosis and treatment, along with dynamic monitoring of the condition, are needed to prevent irreversible injury to joint function. The clinical symptoms of the disease are non-specific, and guidelines recommend that only erythrocyte sedimentation rate (ESR) and C-reactive protein (CRP) level are used as staging indicators. ${ }^{4}$ This makes early and accurate diagnosis and treatment difficult.

Serum amyloid A protein (SAA) is an acute-phase response protein with a short half-life, which shows early and marked elevation. ${ }^{5-7}$ SAA has important diagnostic and therapeutic roles in acute inflammatory disease, and its clinical value in immune diseases has recently been reported. However, there are few reports on the role of SAA in monitoring AS. Therefore, the present study was conducted to observe changes in SAA in the pathogenesis of patients with AS and to investigate the relationship 
between the SAA and AS phase, in order to confirm the clinical value of SAA in the diagnosis and treatment of AS.

\section{Subjects and Methods}

\section{Study Subjects}

Data from 78 patients with AS (the AS group) were collected in our hospital between June 2017 and December 2019. There were 50 males and 28 females, with an average age of $32.64 \pm 13.93$ years (range 10-57 years). All patients met the diagnostic criteria for AS as revised by the Chinese Medical Association Rheumatology Branch in 2010. ${ }^{4}$ Patients with other autoimmune diseases and tumors were excluded. According to the Bath Ankylosing Spondylitis Disease Activity Index (BASDAI) criteria, patients in the AS group were divided into those in the remission phase (36 cases) and those in the active phase (BASDAI grade $\geq 4,42$ cases). During the same time period, 80 subjects who were healthy on physical examination were enrolled as the control group. There were 51 males and 29 females, with an average age of $31.33 \pm 12.16$ years (range 10-58 years). None of these subjects had major disorders of the heart, liver, or kidneys (Figure 1).

\section{Apparatus and Reagents}

SAA was detected by the Hitachi Automatic Biochemistry Analyzer 7600, Japan (Batch number: 200713101). ESR was detected by the ALIFAX (Italy) TEST1 automatic rapid hematocrit meter (Batch number: 04528) and CRP was determined by the Jet-iStar 3000 immunoassay analyzer manufactured by Zhong Han Shengtai Biotechnology Co (Batch number: CRP20101022F).

\section{Detection Methods}

Early in the morning, $5 \mathrm{~mL}$ and $2 \mathrm{~mL}$ samples of venous blood were collected from all participants, after fasting, and placed in a separator gel pro-coagulation tube and an EDTA anticoagulation tube, respectively. The separator gel procoagulation tube was centrifuged at $3000 \mathrm{r} / \mathrm{min}$ for $10 \mathrm{~min}$ for further detection of SAA. The sample in the EDTA anticoagulation tube was used to detect the CRP level and ESR.

\section{Statistical Analysis}

SPSS 22.0 software (IBM) was used for data analysis. The Kolmogorov-Smirnov normality test was used to analyze the normal distribution of the data. Continuous data were expressed as $\bar{x} \pm s$. The $t$-test was used to compare means between groups. Pearson's test was used for correlation analysis. A $p$ value of $<0.05$ was considered statistically significant.

\section{Results}

\section{SAA and CRP Levels and ESR}

SAA and CRP levels and ESR were all significantly higher in the AS group compared with the control group, although the difference in ESR was relatively small (Table 1).

\section{The Diagnostic Efficacy of SAA and CRP Levels and ESR in AS}

Analysis revealed that SAA and CRP levels and ESR had relatively high diagnostic efficacy for AS. Overall, the efficacy of the SAA level was higher than that of the CRP level and ESR, and the efficacy of the CRP level was higher than that of ESR (Table 2).

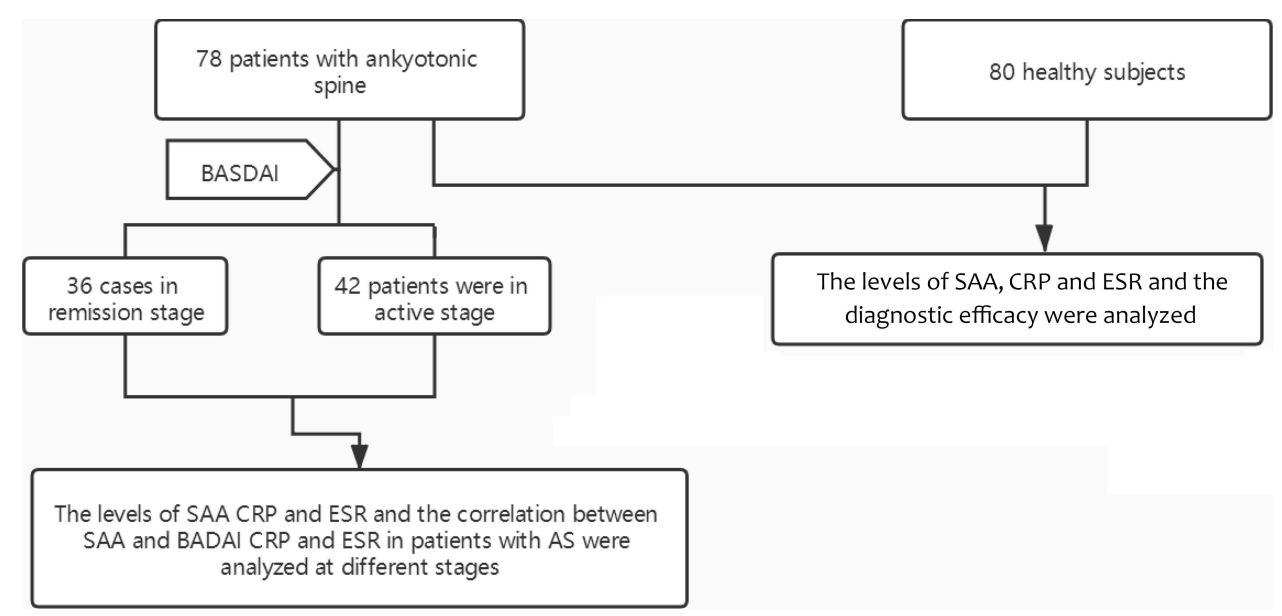

Figure I Case enrollment process. 
Table I Comparison of the Detection Results of SAA, CRP and ESR Between the Two Groups of Subjects

\begin{tabular}{|l|c|c|c|c|}
\hline Group & Number of Case & SAA $(\mathbf{n g} / \mathbf{m L})$ & CRP $(\mathbf{m g} / \mathbf{L})$ & ESR $(\mathbf{m m} / \mathbf{l h})$ \\
\hline AS Group & 78 & $39.65 \pm 12.32$ & $36.12 \pm 14.23$ & $42.03 \pm 12.56$ \\
Control group & 80 & $7.64 \pm 1.32$ & $4.33 \pm 1.54$ & $16.05 \pm 11.01$ \\
$t$ & - & 3.35 & 4.03 & 2.21 \\
$P$ & - & 0.011 & 0.008 & 0.019 \\
\hline
\end{tabular}

Table 2 The Value of SAA, CRP and ESR in the Diagnosis of Ankylosing Spondylitis

\begin{tabular}{|l|c|c|c|c|c|c|}
\hline Item & $\begin{array}{c}\text { Sensitivity } \\
\text { (\%) }\end{array}$ & $\begin{array}{c}\text { Specificity } \\
\text { (\%) }\end{array}$ & $\begin{array}{c}\text { Positive Predictive Value } \\
\text { (\%) }\end{array}$ & $\begin{array}{c}\text { Negative Predictive Value } \\
\text { (\%) }\end{array}$ & $\begin{array}{c}\text { Youden Index } \\
\text { (\%) }\end{array}$ & $\begin{array}{c}\text { Consistency } \\
\text { (\%) }\end{array}$ \\
\hline SAA & 79.49 & 77.50 & 77.50 & 77.50 & 56.99 & 78.48 \\
CRP & 64.10 & 87.50 & 83.33 & 87.50 & 51.60 & 75.95 \\
ESR & 61.54 & 85.00 & 80.00 & 85.00 & 46.54 & 73.42 \\
\hline
\end{tabular}

Changes in SAA and CRP Levels and ESR in AS Patients by Disease Stage

SAA and CRP levels and ESR in AS patients in the active phase were significantly higher than in patients in the remission phase, as shown in Table 3.

\section{Correlation Between SAA Level and BASDAI Grade, CRP Level, and ESR}

Correlation analysis revealed that the SAA level was positively correlated with BASDAI grade, the CRP level, and ESR, as demonstrated in Table 4.

\section{Discussion}

AS is a chronic, progressive and inflammatory disease of unknown etiology. Most researchers believe that it is caused by a combination of infectious, autoimmune, genetic, environmental, and other factors. In addition, the insidious onset of AS and the lack of specific symptoms make it difficult to diagnose and treat. This leads to under- and misdiagnosis and causes great suffering to patients. ${ }^{8-10}$ Therefore, it is important to find convenient and reliable laboratory indicators for the early diagnosis and management of AS. CRP level and ESR are two established diagnostic indicators given in guidelines for AS, but, as broad-spectrum inflammatory factors, their low sensitivity dictates that these two indicators cannot be used alone in the monitoring of AS. Instead, they should be used in combination with other indicators with higher sensitivity. ${ }^{4} \mathrm{SAA}$ is a non-specific acute phase-response protein that has recently been found to be elevated in patients with immune diseases involving chronic inflammation, and it has considerable potential value, especially in the assessment of joints affected by rheumatoid disease. ${ }^{11}$ The expression of SAA is abnormal in patients with multiple immune diseases, particularly when the disease is in an active phase, but the clinical significance of this is not conclusive. Changes in SAA levels in patients with AS have been reported relatively rarely and, thus, should be investigated for their potential use in diagnosis, treatment, and monitoring of the disease.

In the present study, SAA and CRP levels and ESR were significantly higher in the AS group than in the healthy controls. Li et $\mathrm{al}^{12}$ also reported significantly higher levels of SAA in patients with AS, but this finding needs to be verified by further studies. The sensitivity of SAA level as a diagnostic tool for AS was $79.49 \%$, which was significantly higher than that of the CRP level (64.10\%) and ESR (61.54\%). At $77.50 \%$, the specificity

Table 3 Comparison of the Detection Results Between AS in the Remission Phase and Active Phase

\begin{tabular}{|l|c|c|c|c|}
\hline Item & Number of Case & SAA $(\mathbf{n g} / \mathbf{m L})$ & CRP (mg/L) & ESR (mm/Ih) \\
\hline Active phase & 42 & $56.18 \pm 17.25$ & $58.31 \pm 20.25$ & $65.18 \pm 21.65$ \\
Remission phase & 36 & $20.36 \pm 5.36$ & $10.23 \pm 3.65$ & $15.02 \pm 5.36$ \\
$t$ & - & 2.98 & 4.95 & 4.62 \\
$P$ & - & 0.015 & 0.001 & 0.009 \\
\hline
\end{tabular}


Table 4 Correlation Between SAA and BASDAI, CRP and ESR

\begin{tabular}{|l|c|c|c|c|c|c|}
\hline \multirow{2}{*}{ Item } & \multicolumn{2}{|c|}{ BASDAI } & \multicolumn{2}{c|}{ CRP $(\mathbf{m g} / \mathbf{L})$} & \multicolumn{2}{c|}{ ESR $(\mathbf{m m} / \mathbf{l} \mathbf{h})$} \\
\cline { 2 - 7 } & $\mathbf{r}$ & $\boldsymbol{P}$ & $\mathbf{r}$ & $\boldsymbol{P}$ & $\mathbf{r}$ & $\boldsymbol{P}$ \\
\hline SAA $(\mathrm{ng} / \mathrm{mL})$ & 0.77 & 0.005 & 0.68 & 0.011 & 0.62 & 0.012 \\
\hline
\end{tabular}

of the SAA level was slightly lower than that of the CRP level $(87.50 \%)$ and ESR $(85.00 \%)$. However, both the Youden index and the concordance of the SAA level were higher than those of the CRP level and ESR. These results not only confirm the high efficacy of the SAA level in the diagnosis of AS, but also suggest that it is more effective than either the CRP level or ESR. This may be due to the fact that the SAA level starts to rise after approximately $8 \mathrm{~h}$ of inflammatory response, even in mild infections, whereas significant elevations of the CRP level and ESR are generally only seen in bacterial infections.

The present study also confirms that the SAA level was increased in both the remission and active phases in patients with AS. Further comparisons of SAA and CRP levels and ESR revealed that all three indicators were substantially higher in the active phase than in the remission phase. This indicates that SAA, like established indicators such as the CRP level and ESR, might be correlated with disease activity in AS. AS is a chronic inflammatory disease in which mononuclear macrophages produce a variety of inflammatory responsive cytokines, such as IL-1, IL-6, and TNF, during the active phase. ${ }^{13,14}$ When cytokines are stimulated, the synthesis of SAA is increased in the liver. In addition, a particularly important property of SAA is the ability of degradation products to be deposited in different organs in the form of amyloid A protofibrils, which may lead to chronic inflammatory disease. This pathophysiological process might be an important reason for the substantial elevation of SAA during the active phase and slight elevation during the remission phase in patients with AS. ${ }^{15}$

HLA-B27 is strongly associated with the development of AS and is an established indicator of the disease, but its role in monitoring and dynamic observation is minimal. Guidelines for the diagnosis and treatment of AS recommend only the CRP level and ESR as indicators for monitoring disease activity. The BASDAI is the most widely used system for evaluating the activity of AS. ${ }^{16}$ In this study, SAA and CRP levels and ESR were strongly and positively correlated with BASDAI grade, and the correlation between the SAA level and the BASDAI grade was significantly stronger than those between the BASDAI grade and the CRP level or ESR. These results further verify that the SAA level is a better indicator for monitoring disease activity in patients with AS than the CRP level and ESR.

In summary, the SAA level is a potentially reliable laboratory indicator not only for the diagnosis and management of AS, but also for the assessment of disease activity and prognosis. Therefore, it could be used as an adjunct to the clinical management of AS.

\section{Ethics Approval}

This study was conducted in accordance with the declaration of Helsinki. This study was conducted with approval from the Ethics Committee of The First People's Hospital of Yuhang District, Hangzhou. All participants provided informed consent to participate, except for in the case of participants under the age of 18 , in which case informed consent was provided by a parent or legal guardian.

\section{Disclosure}

All authors have contributed significantly to the manuscript and declare that the work is original and has not been submitted or published elsewhere. None of the authors have any financial disclosure or conflicts of interest.

\section{References}

1. Law L, Beckman Rehnman J, Deminger A, Klingberg E, Jacobsson LTH, Forsblad-d'Elia H. Factors related to health-related quality of life in ankylosing spondylitis, overall and stratified by sex. Arthritis Res Ther. 2018;20(1):284. doi:10.1186/s13075-018-1784-8

2. Lories RJ, Haroon N. Evolving concepts of new bone formation in axial spondyloarthritis: insights from animal models and human studies. Best Pract Res Clin Rheumatol. 2017;31(6):877-886. doi:10.1016/j.berh.2018.07.007

3. $\mathrm{Lu} \mathrm{MC}$, Huang $\mathrm{KY}$, Tung $\mathrm{CH}$, et al. Factors associated with disease-specific quality of life in Taiwanese patients with ankylosing spondylitis: a cross-sectional study. BMJ Open. 2019;9(6):e28966. doi:10.1136/bmjopen-2019-028966

4. Rheumatology Society of Chinese Medical Association. Guidelines for diagnosis and treatment of ankylosing spondylitis. Chin J Rheumatol. 2010;14(8):557-559.

5. Wakai M, Hayashi R, Tanaka S, et al. Serum amyloid A is a better predictive biomarker of mucosal healing than $\mathrm{C}$-reactive protein in ulcerative colitis in clinical remission. BMC Gastroenterol. 2020;20 (1):85. doi:10.1186/s12876-020-01229-8

6. Zhou H, Chen M, Zhang G, Ye RD. Suppression of lipopolysaccharide-induced inflammatory response by fragments from serum amyloid A. J Immunol. 2017;199(3):1105-1112. doi:10.4049/jimmunol.1700470 
7. Facci L, Barbierato M, Zusso M, Skaper SD, Giusti P. Serum amyloid A primes microglia for ATP-dependent interleukin-1 $\beta$ release. J Neuroinflammation. 2018;15(1):164. doi:10.1186/s12974-018-1205-6

8. Jamalyaria F, Ward MM, Assassi S, et al. Ethnicity and disease severity in ankylosing spondylitis a cross-sectional analysis of three ethnic groups. Clin Rheumatol. 2017;36(10):2359-2364. doi:10.1007/s10067-017-3767-6

9. Hong C, Kwan YH, Leung YY, Lui NL, Fong W. Comparison of ankylosing spondylitis and non-radiographic axial spondyloarthritis in a multi-ethnic Asian population of Singapore. Int J Rheum Dis. 2019;22(8):1506-1511. doi:10.1111/1756-185X.13603

10. Jones GT, Mallawaarachchi B, Shim J, Lock J, Macfarlane GJ. The prevalence of fibromyalgia in axial spondyloarthritis. Rheumatol Int. 2020;40(10):1581-1591. doi:10.1007/s00296-020-04621-5

11. Laboratory Medicine Committee of Chinese Association of Integrated Traditional and Western Medicine. The clinical application of serum amyloid A in infectious diseases. Chin J Lab Med. 2019;42 (3):186-192

12. Li FY, Lv Z. Clinical significance of serum SAA and MMP-3 levels in patients with ankylosing spondylitis. Clin Res. 2018;26(6):44-45.
13. de Vries MK, van Eijk IC, van der Horst-bruinsma IE, et al. Erythrocyte sedimentation rate, C-reactive protein level, and serum amyloid a protein for patient selection and monitoring of anti-tumor necrosis factor treatment in ankylosing spondylitis. Arthritis Rheum. 2009;61(11):1484-1490. doi:10.1002/art.24838

14. Jung SY, Park MC, Park YB, Lee SK. Serum amyloid a as a useful indicator of disease activity in patients with ankylosing spondylitis. Yonsei Med J. 2007;48(2):218-224. doi:10.3349/ymj.2007.48.2.218

15. Fellahi S, Béraud L, Capeau J, et al. Validation des valeurs usuelles proposées par un fournisseur sans accès à une population témoin: exemple de la sérum amyloïde A [Validation of the usual values provided by a supplier without access to a control population: example of serum amyloid A]. Ann Biol Clin (Paris). 2019;77(3):255-259. doi:10.1684/abc.2019.1439

16. Li DX, Xu SQ, Wu Y, et al. Comparison of BASDAI and ASDAScrp in a measure of disease activity in patients with spondyloarthropathy. Gen Pract Res. 2017;15(1):11-12.
International Journal of General Medicine

\section{Publish your work in this journal}

The International Journal of General Medicine is an international, peer-reviewed open-access journal that focuses on general and internal medicine, pathogenesis, epidemiology, diagnosis, monitoring and treatment protocols. The journal is characterized by the rapid reporting of reviews, original research and clinical studies
Dovepress

across all disease areas. The manuscript management system is completely online and includes a very quick and fair peer-review system, which is all easy to use. Visit http://www.dovepress.com/ testimonials.php to read real quotes from published authors. 